\title{
Electron mobility and localization in dense $\mathrm{He}^{4}$ gas
}

\author{
Harold R Harrison $†$, Leonard M Sander and BE Springett \\ Department of Physics, The University of Michigan, Ann Arbor, Michigan 48104
}

MS received 13 November 1972

\begin{abstract}
An extensive experimental investigation has been carried out on the mobility of electrons in dense helium gas with particular emphasis on the drastic decreases in mobility which are believed to result from the onset of electron localization in the fluid. The mobility was measured for gas densities $2<\rho<40 \times 10^{20}$ atoms $\mathrm{cm}^{-3}$, and for gas temperatures $4<T<300 \mathrm{~K}$.

Refinements of former treatments of electron localization via the bubble model are presented which demonstrate a strong correlation between the mobility 'edge' and a localization criterion. Detailed agreement between theory and experiment is not realized, but the general features of the density and temperature dependence of the mobility are reproduced in a consistent manner.
\end{abstract}

\section{Introduction}

The 'bubble' model of the negative carrier in liquid and dense gaseous helium has been thoroughly studied (Kuper 1959, Jortner et al 1965, Levine and Sanders 1967, Springett et al 1967). In this model an excess electron is pictured as inducing a density variation in the fluid through the repulsive electron-helium atom interaction; the density variation is often idealized as a void (the bubble) in which the electron can be self-consistently trapped. The trapping of the carrier drastically decreases its mobility; indeed, it is thought that one of the dramatic manifestations of bubble formation is the "mobility collapse' of electrons in helium gas first discovered by Levine and Sanders (1967). The 'collapse' is the observed decrease of electron mobility by a factor of $10^{4}$ when the gas density increases by a factor of 2 , under proper conditions. The phenomenon is not peculiar to helium (Springett et al 1968).

The bubble model provides a ready explanation of this phenomenon. Bubbles can only be formed under proper conditions of temperature and density (Springett et al 1968). In particular, at constant temperature there is a critical density below which bubbles are not stable. The mobility collapse then can be viewed as the result of the onset of localization of electrons; as the critical density is exceeded electrons begin to be trapped in bubbles and the observed mobility decreases.

Recently an alternate explanation of the collapse has been proposed (Neustadter and Coopersmith 1969, Eggarter and Cohen 1970). Work on electron mobility in systems of fixed but random scattering centres (as in an amorphous semiconductor, for example) led to the prediction that localization of the carrier can occur in traps arising from static density variations in the material. This localization also results in a mobility collapse.

$\uparrow$ Present address: Materials Science and Metallurgical Engineering Department, Purdue University, West Lafayette, Indiana, 47906. 
Unfortunately, both the simple bubble and random scattering theories give predictions for the mobility which agree only qualitatively with experiment. An even more recent treatment of the mobility collapse has attempted to combine certain features of the bubble model with the random scattering theory (Eggarter and Cohen 1971, Eggarter 1972). Good agreement between theory and experiment was obtained with the introduction of certain assumptions. We will discuss this treatment further below.

In the present work we have attempted to throw more light on the systematics of the negative carrier mobility by making mobility measurements over a wide range of density and temperature. We hope these data will aid in the formulation of a more complete description of the collapse. The experimental details and results are presented in $\S 2$. In $\S 3$ we attempt a brief discussion of our results in terms of an improved version of the bubble model. Though a more sophisticated treatment such as that of Eggarter (1972) would clearly be desirable, we consider it worthwhile to continue to use the older theory for two reasons: the bubble model is a well-defined theory with no adjustable parameters which gives as we shall see, a good qualitative description of the mobility collapse. And, pushing the model to its limits seems worthwhile to explicate its deficiencies and strengths. In $\S 4$, we summarize our results.

\section{Experimental results}

The mobility data were obtained using a single gate velocity spectrometer with drift regions of different lengths. This is because we needed to measure mobilities which ranged from $\mu \approx 2 \times 10^{4}$ to $\mu \approx 0.1 \mathrm{~cm}^{2} \mathrm{~V}^{-1} \mathrm{~s}^{-1}$. The electron source was a heavily gold-plated $\beta^{-}$emitter (tritium). The gold-plating was necessary to shorten the range of $\beta^{-}$at low densities. A scale drawing of the experimental cell is shown in figure 1 . The large distance between $S$ and $G_{1}$ is to ensure that only thermal electrons arrive at $\mathrm{G}_{1}$ at even the lowest densities. This distance and the gold thickness on $\mathrm{S}$ were arrived at by calculating the expected range of the $\beta^{-}$from the tritium. Later, it was checked that the calculations were correct by imposing a driving field between $G_{1}$ and the collector and having no field between $S$ and $G_{1}$. Only at densities less than $5.5 \times 10^{19} \mathrm{~cm}^{-3}$ was a current observed under these conditions which agrees well with the calculated value of $4.5 \times 10^{19} \mathrm{~cm}^{-3}$. The cell is operated by using various combinations of $G_{1}, G_{2}, G_{3}$ as a drift space to which a variabie amplitude square-wave is applied. Between the source and the grid to which the square-wave is applied a constant field, equal to that in the drift space, is maintained. This field is floated on the squarewave. Fields and current levels were always such that we were far from space-charge limited conditions. $\mathrm{G}_{4}$ and the collector, which is essentially at ground potential, formed a final constant field region. The mobility is found from curves of current versus square-wave frequency. The frequency was swept in time at a slow rate using a HP3300A function generator, and the current-frequency curves displayed on an HP Moseley 2FA $x-y$ chart recorder. The frequency axis was calibrated each 5 sweeps with a HP 5245L counter. The current detector was an Analog Devices $310 \mathrm{~J}$ operational amplifier with a minimum sensitivity of $\sim 10^{-14}$ amps.

The current-frequency relation is

$$
\begin{aligned}
I(v) & =\frac{1}{2} I_{0}\left(l-v / v_{\mathrm{c}}\right) & & v<v_{\mathrm{c}} \\
& =0 & & v \geqslant v_{\mathrm{c}}
\end{aligned}
$$




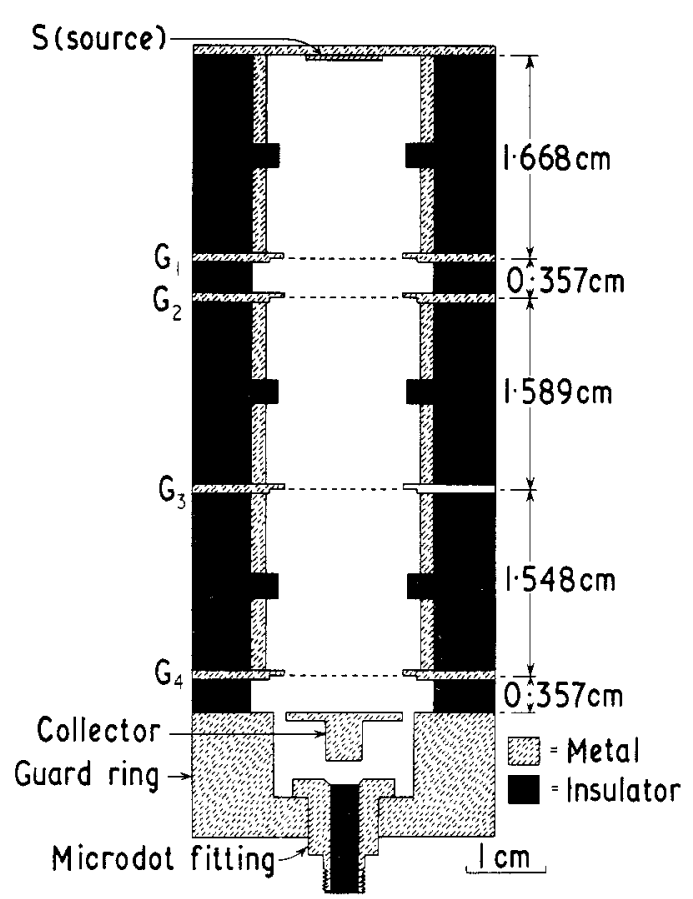

Figure 1. Scale drawing of measurement cell. The metal is gold plated brass. The insulator is nylon. The grids are gold plated copper with $82+2 \%$ transmission, 100 lines/inch. Total shrinkage of the cell on cooling is estimated at $0.5 \%$.

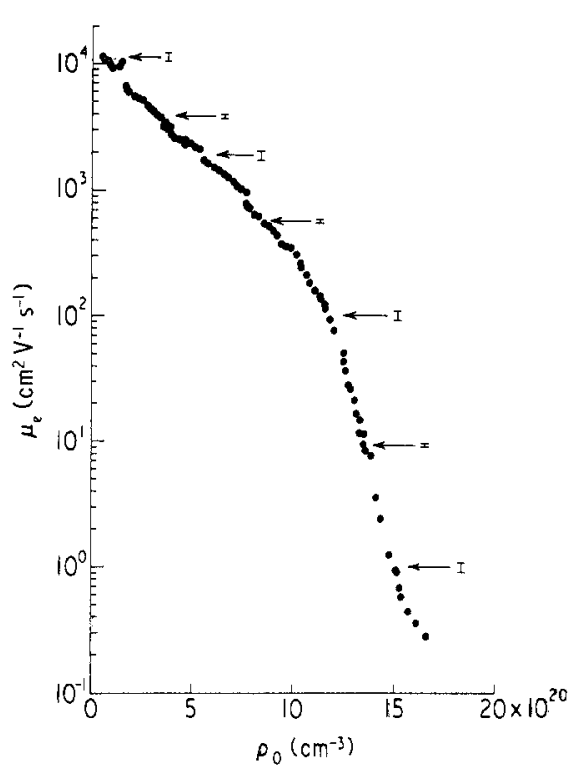

Figure 2. Electron mobility versus number density at $4.18 \mathrm{~K}$, showing scatter in the data. The error bars are representative of the errors in determining $v_{c}$ in their respective ranges of the graph.

where

$$
v_{\mathrm{c}}=\mu V_{\mathrm{i} 4} / 2 d_{\mathrm{i}_{4}}^{2}
$$

$V_{\mathrm{i}_{4}}$ is the voltage imposed across the drift space, and $d_{\mathrm{i}_{4}}$ is the physical length of the drift space. With this particular cell we were able to make measurements up to about $50 \mathrm{kHz}$ and down to about $3 \mathrm{~Hz}$, although most measurements fell in the range 10 to $10^{4} \mathrm{~Hz}$.

Temperature control is effected by maintaining the copper case surrounding the measuring cell in equilibrium with $\mathrm{He}^{4}$ at its boiling point by means of a cold finger around which is wound a $100 \Omega$ heater. An auxiliary heater was wrapped around the can itself. Temperature could be maintained constant to within $0.05 \mathrm{~K}$ for periods of 10 to 15 minutes with this arrangement, which is about 5 times as long as the time for a single frequency sweep. Additional details of the experimental procedure can be found in Harrison (1971).

Standard techniques were used to keep the experimental cell and working gas clean. Pressure was measured with Bourdon-type gauges to an accuracy of $2 \%$ and temperature with a $\mathrm{Ge}$ thermometer, calibrated against $\mathrm{N}_{2}, \mathrm{He}^{4}$, and $\mathrm{H}_{2}$ vapour pressure, to an accuracy of $\frac{1}{2} \%$. The density was obtained from the $P-T$ measurements using the virial equation of state. Thus the density measurements are accurate to $2 \frac{1}{2} \%$. There are two major sources of error. The first is a systematic error of $\pm 5 \%$ due to the fact 
that the electrical length of the drift space does not precisely coincide with the physical length. The second comes from errors in determining $v_{c}$, or equivalently, the frequency at which the collected current falls to zero. This error is variable due to such things as electrical noise, mechanically induced noise, and the fact that the HP 3300A is arranged in decades of frequency. Figure 2 shows discrete data points, together with error bars originating from the determination of $v_{c}$, but not including systematic error, which remains constant for all points. Further data curves do not show discrete points or error bars in the interests of clarity.

The mobility measurements themselves have an absolute accuracy of $\pm 10 \%$, half of this being geometrical in origin. In a given run the internal accuracy is $\pm 5 \%$, this being the maximum error and occurs at low density when very small fields must be used, and high density when the signal is weak.

We present smoothed plots of our data in figures 3 and 4 . Figure 3 is measured mobility versus density at constant $\mathrm{T}$; the temperatures are marked on the curves.

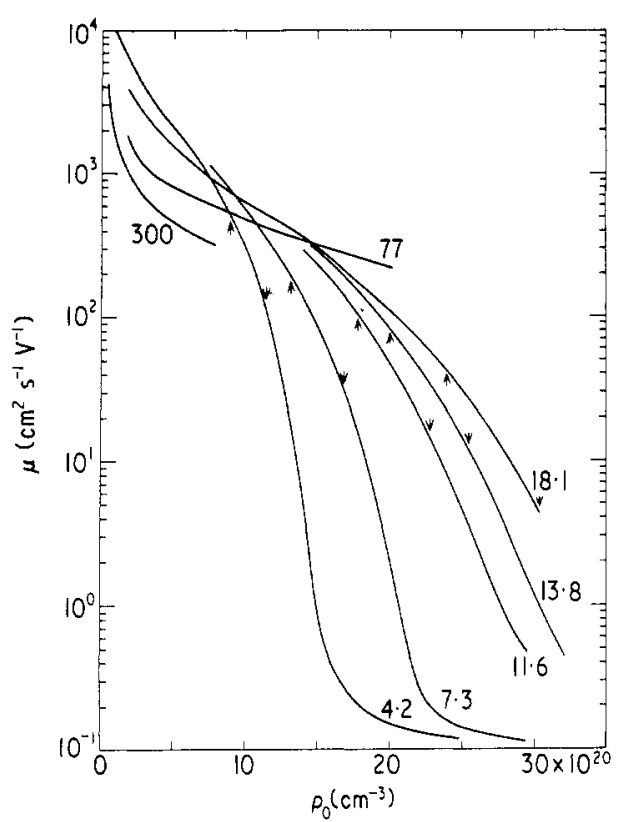

Figure 3. Electron mobility versus density in helium-4. The temperature at which the data were taken are marked on the curves in degrees Kelvin. (The arrows are explained in $\S 4$.)

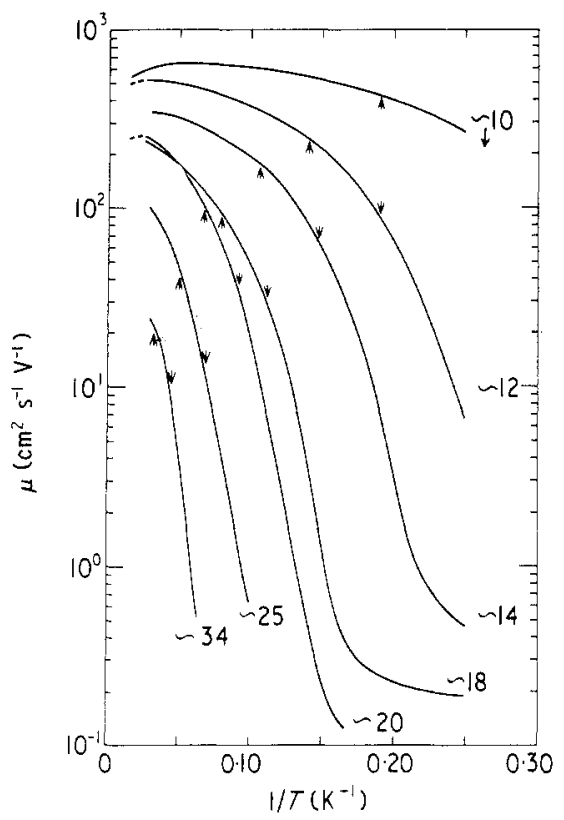

Figure 4. Electron mobility versus inverse temperature at nearly constant density in helium-4. The density is marked on the curves in units of $10^{20} \mathrm{~cm}^{-3}$. (The arrows are explained in $\$ 4$.)

Figure 4 is measured mobility versus $T^{-1}$ at almost constant density, which is marked on the curves in units of $10^{20} \mathrm{~cm}^{-3}$. The density decreases by about $10 \%$ on each of these curves as $T$ increases because of a finite volume at room temperature. For a more detailed presentation of the data see Harrison (1971). The $\mu$ versus $\rho$ curve at $300 \mathrm{~K}$ agrees with the measurements of Grunberg (1968) to within $5 \%$, and the curve at $4.2 \mathrm{~K}$ agrees with the measurements of Levine and Sanders (1967) to within $10 \%$. (The significance of the arrows in the figures will be explained below.) Each curve in 
the figures is obtained from some sixty to a hundred data points. Figure 5 is an alternative presentation of some of the data displayed as the $(\rho, T)$ coordinates of the equation $\mu / \mu_{\mathrm{f}}=$ constant. The points are taken from both constant density and constant temperature runs; thus the internal consistency of the data is quite good. The solid line corresponds to the upward arrows in figures 3 and 4. Its significance will be explained later. $\mu_{\mathrm{f}}$ is defined in equation (9).

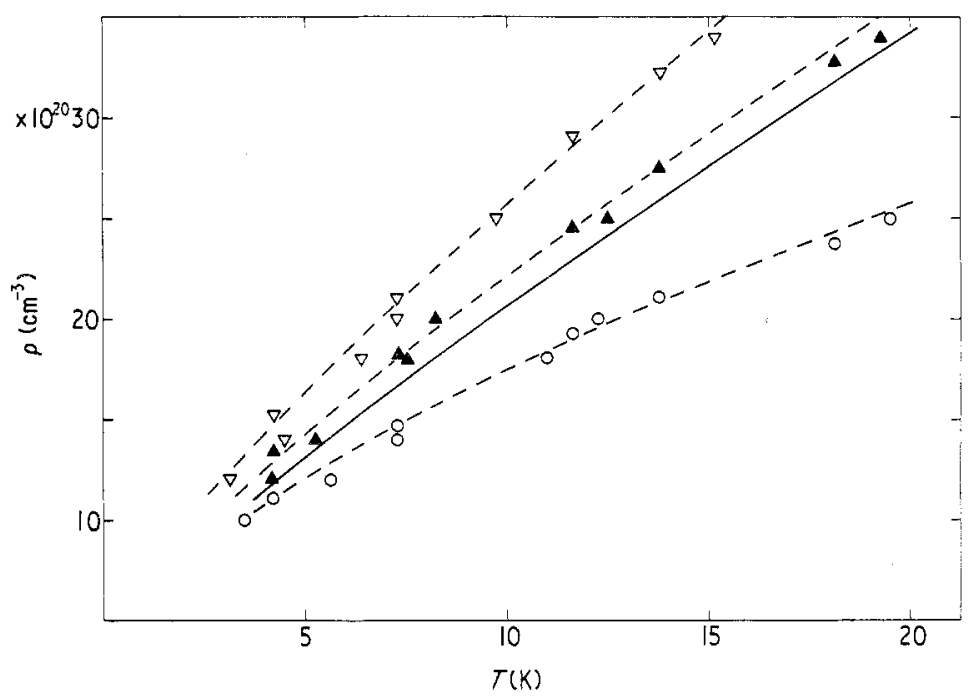

Figure 5. Plot of $(\rho, T)$ coordinates for which $\mu / \mu_{\mathrm{f}}=$ constant. $\bigcirc$ constant $=0 \cdot 1$ : constant $=0.01 ; \nabla$ constant $=0.001$. The dashed lines are a guide to the eye. The solid line divides the $(\rho, T)$ plane into a region where bubbles are unstable (below the line) and where bubbles are stable according to the rounded-well model. For constant $=1.0$, the curves would be described by $\rho T^{1 / 2}=$ constant, in the low-density limit.

It is pertinent at this point to make a few general comments about these results. First, although not shown in figure 3 the shape of the curvature of $\mu$ versus $\rho$ initially follows the dilute gas dependence on density, being roughly proportional to $1 / \rho$. We interpret this to mean that the electron is not localized at low densities, and may acquire an effective mass and scattering length weakly dependent on density. Second, the high density flat portion of the figure can be explained in terms of a viscous limited mobility; the moving object must have a radius of $\sim 15 \AA$.

The region over which the largest drop in mobility occurs is both density and temperature dependent, covering a larger range of density, the higher the temperature. If one looks at figure 4 it can be seen that over part of the range $\mu \alpha \exp (-\Delta / T)$, with $\Delta$ dependent on $\rho$. Since there is always some curvature, $\Delta$ is also dependent on $T$. This feature we interpret as being due to the onset of electron localization, the binding energy of the trap depending on $\rho$ and $T$. Lastly, the data curve in figure 4 for $\rho \sim 10^{21}$ displays a maximum which indicates that the electron is tending to change from being quasi-free to quasi-localized at this temperature. In figure 5 the $\rho$-axis is closely proportional to the average penetration barrier of electrons into the fluid, $V_{0}$. The $T$-axis is proportional to the average thermal energy of the He atoms. It can be seen then that $\mu / \mu_{\mathrm{f}}=$ constant is roughly equivalent to $V_{0} / k T=$ constant, again indicating that localization is the mechanism responsible for the mobility decrease. 


\section{Theory}

The problem of electron localization due to bubble formation in dense helium has been discussed extensively in the literature. In this section we will refine the previous treatments of the model in an attempt to fit the data. As we will see, a modest improvement results.

Our basic description of an electron in dense helium gas is a slightly modified version of the earlier theories. We adopt a repulsive pseudopotential

$$
V(\boldsymbol{r}-\boldsymbol{R})=A \delta(\boldsymbol{r}-\boldsymbol{R})
$$

to represent the interaction of an electron at $\boldsymbol{r}$ with an atom at $\boldsymbol{R}$. Thus $V_{0}=A \rho_{0}$ is the average barrier for penetration of electrons into the fluid when the measured density is $\rho_{0}$. We take $V_{0}$ from the Wigner-Seitz calculations of Jortner et al (1965). The electron will be taken to be governed by the effective Schrödinger equation,

$$
\frac{\hbar^{2}}{2 m} \nabla^{2} \psi+A \rho(\boldsymbol{r}) \psi=E_{\mathrm{e}} \psi .
$$

Here $\rho(\boldsymbol{r})$ is the gas density, which may vary in space. This equation plays the role of an effective-mass equation with $\psi$ an envelope wavefunction. Equation (2), together with the equation of state and the condition of hydrostatic equilibrium,

$$
p / \rho=-\nabla\left(A|\psi|^{2}\right)
$$

form a closed set which could be solved self-consistently. An attempt along these lines has been made by Levine and Sanders (1967), and for a similar system by Clark (1965), and by Harrison (1971) using the $\rho(\boldsymbol{r})$ of equation (6) below, as a first approximation.

Most treatments of the bubble problem have been content to solve this set of equations only approximately. A popular model, the so-called square well (Springett et al 1967), fixes the density as zero within a certain radius $r_{0}$, and a constant outside. The total free energy is then minimized with respect to the parameter $r_{0}$. We have attempted to use more general density profiles, consistent with a low density system, to improve the accuracy.

The motivation for this work was our conviction that in a gas the square well prescribed a density variation that was much too abrupt. (The situation in the liquid is such that the square well is a good approximation.) We investigated the free energy, $F$, of the system of electron plus fluid in the presence of an arbitrary density variation $\rho(\boldsymbol{r})$. We use the approximation,

$$
F=F_{0}+E_{\mathrm{e}}+F_{\mathrm{g}}
$$

where $F_{0}$ is the free energy of the undisturbed gas, $E_{\mathrm{e}}$ is obtained from equation (2), and $F_{\mathbf{g}}$ is the extra free energy of the gas due to the enforced density variation.

It is easy to derive an expression for $F_{\mathrm{g}}$ in terms of the density $\rho(\boldsymbol{r})$,

$$
\begin{aligned}
& \left.F_{\mathrm{g}}=\rho_{0} k_{\mathrm{B}} T \int \mathrm{d}^{3} \boldsymbol{r}\left\{(1-g(\boldsymbol{r}))+B_{1} \rho_{0}(1-g(\boldsymbol{r}))^{2}+g(\boldsymbol{r}) \ln g(\boldsymbol{r})\right)\right\} \\
& g(\boldsymbol{r})=\rho(\boldsymbol{r}) / \rho_{0} .
\end{aligned}
$$

Here $\rho_{0}$ is the limiting value of the gas density far from the electron, and $B_{1}$ is the first viral coefficient. 
We chose first a smoothed spherically symmetric form for the function $\rho(\boldsymbol{r})$ (or alternatively, $g(\boldsymbol{r}))$

$$
g(\boldsymbol{r})=1-1 / \cosh ^{2}\left(\boldsymbol{r} / \boldsymbol{r}_{0}\right) .
$$

The peculiar form of this 'rounded-well' approximation has been chosen so that the Schrödinger equation (2) is exactly soluble (Landau and Lifshitz 1950). It is straightforward to minimize $F$ with respect to $r_{0}$. (Details of the calculation may be found in Harrison 1971.) In table 1 we give the calculated values of $\Delta F=F-F_{0}-V_{0}$ for $T=3.96 \mathrm{~K}$, along with similar values for the square well model.

Table 1. Free energy divided by $k_{\mathrm{B}} T$ as a function of density for two versions of the bubble model, for $T=3.96 \mathrm{~K}$

\begin{tabular}{lrrrr}
\hline$\rho_{0} \times 10^{-20}\left(\mathrm{~cm}^{-3}\right)$ & \multicolumn{2}{c}{ Rounded well } & \multicolumn{2}{c}{ Square well } \\
\cline { 2 - 5 } & $\Delta F / k_{\mathrm{B}} T$ & $r_{0}(\AA)$ & $\Delta F / k_{\mathrm{B}} T$ & $r_{0}(\AA)$ \\
\hline 9.0 & 5.20 & 19.37 & $-\dagger$ & - \\
9.5 & 4.33 & 18.83 & - & - \\
10.0 & 3.66 & 18.34 & - & - \\
10.5 & 1.77 & 17.85 & - & - \\
11.0 & 0.12 & 17.41 & - & - \\
11.5 & -1.58 & 17.00 & 16.47 & 19.18 \\
12.0 & -3.39 & 16.62 & 14.02 & 19.70 \\
12.5 & -5.27 & 16.27 & 11.50 & 20.10 \\
13.0 & -7.23 & 15.92 & 9.19 & 20.30 \\
13.5 & -9.59 & 15.60 & 6.88 & 20.50 \\
\hline
\end{tabular}

$\leftarrow$ The dashes indicate that no stable bubble is predicted.

The variational parameter $r_{0}$ is the bubble radius at these values of density and temperature.

Below $\rho_{0}=9 \times 10^{20} \mathrm{~cm}^{-3}$ no minimum of $F\left(r_{0}\right)$ exists: this indicates that the bubble is not stable. Note that for all values of the density the free energy is smaller for the rounded-well model than for the square well.

The form of equation (6) was chosen entirely for algebraic convenience. If our equations are solved self-consistently, the form of the density profile around the electron becomes squarer and the density at the origin does not go to zero. We attempted to make a more rational choice by using a density profile which yields the Woods-Saxon potential familiar in nuclear physics:

$$
g(r)=\left[1+\exp \left\{-\left(r-r_{1}\right) / r_{0}\right\}\right]^{-1} .
$$

In this expression $r_{0}$ and $r_{1}$ are both free parameters. We allow both the size of the well and its 'roundedness' to vary: as $r_{1} \rightarrow 0, g(\boldsymbol{r})$ approaches the form appropriate for a square well. Thus we can directly obtain some measure of the optimum shape of the well.

To calculate $F$ in this case we solved equation (2) on a computer for a large number of values of $r_{0}$ and $r_{1}$, and also evaluated the integrals in equation (5). We find that the well never becomes particularly 'square' even for rather high values of density when the bubble state is extremely favourable thermodynamically. It is worthy of note that bubbles are mechanically stable before they are thermodynamically stable, which suggests that under certain circumstances one could observe two distinct mobilities. 
Such a situation has in fact been observed in gaseous $\mathrm{H}_{2}$ (Harrison and Springett 1971a, b).

To calculate the mobility we use the phenomonological equation given by several authors (for example, Young 1970),

$$
\mu=\frac{\mu_{\mathrm{b}}+\mu_{\mathrm{f}} \exp \left(\Delta F / k_{\mathrm{B}} T\right)}{1+\exp \left(\Delta F\left(k_{\mathrm{B}} T\right)\right.} .
$$

Here the total mobility $\mu$ is expressed in terms of the mobility of the bubble, $\mu_{\mathrm{b}}$, and of the free electron, $\mu_{\mathrm{f}} . \Delta F$ is the free energy difference between the bubble state and the free state.

For $\mu_{\mathrm{f}}$ we use the expression of Levine and Sanders (1967)

$$
\mu_{\mathrm{f}}=\frac{4 e}{3 \rho_{0} \sigma\left(2 \pi m k_{\mathrm{B}} T\right)^{1 / 2}}\left(1+2 B_{1} \rho_{0}\right)
$$

Here $e$ and $m$ are electronic charge and mass and $\sigma$ is the cross section for electronhelium scattering, which we take to be $4.9 \times 10^{-16} \mathrm{~cm}^{2}$ (O'Malley 1963).

For the bubble we use the interpolation scheme of Levine and Sanders (1967). An example of the derived mobility is plotted as a function of density in figure 6 .

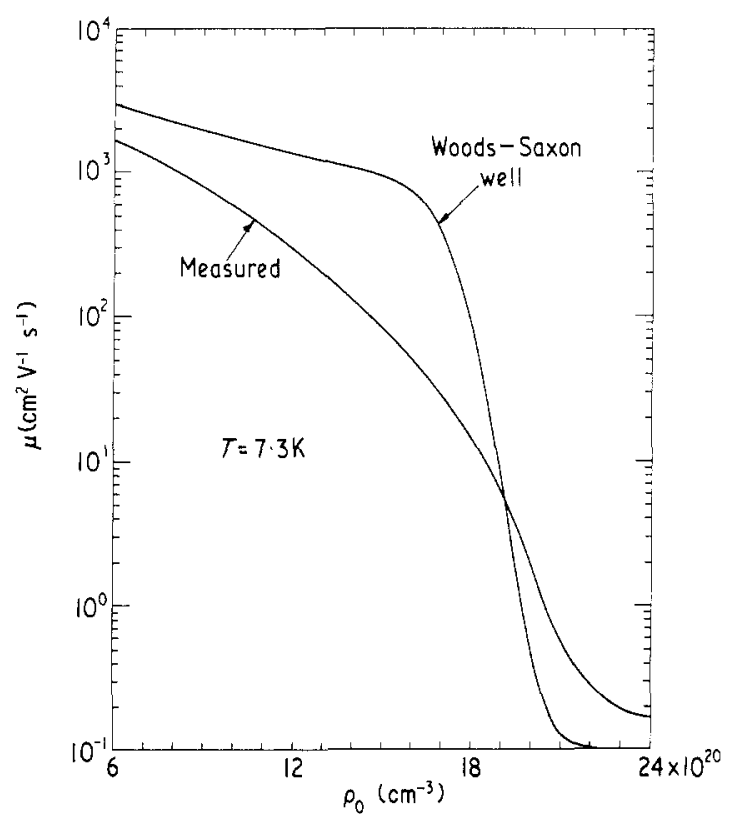

Figure 6. Comparison of the Woods-Saxon model (equation (10)) with experiment for the density dependence of the mobility at $T=7.30 \mathrm{~K}$.

The fit is not terribly good as we should expect since the expression for $\mu_{\mathrm{f}}$, equation (9), only takes into account the first order correction due to fluctuations. Eggarter has examined the problem in depth and has produced an adequate fit to the data, using two free parameters. However, our aim is not so much to fit the data closely, as to demonstrate the importance of the role of electron localization in causing the mobility collapse. It is interesting that the rounded-well predictions do not differ greatly from 
those of the optimized Woods-Saxon potential. It would be desirable to include a realistic treatment of the localized state in the procedure used by Eggarter, and it would seem that the rounded-well could be a convenient and tractable substitute for a selfconsistent solution.

\section{Discussion}

It is readily apparent that the approximate equation (8) gives a fair account of the mobility variation. The rounded-well or the Woods-Saxon models locate the transition in a general way. This point is emphasized in figures 3 and 4 where the small upward arrows indicate the density or temperature at which localization first occurs for the rounded well. The positions of these arrows for the Woods-Saxon model are shifted slightly to the left. For comparison, the downward arrows show the prediction of the square-well model. Considering the wide range of density and temperature covered, and that the model contains no adjustable parameters, it is difficult to believe that the systematic correspondence between predictions of localization and decrease in mobility is coincidence.

Figure 5 shows this correspondence even more clearly. The solid line corresponds to those points in the $(\rho, T)$ plane where the rounded-well bubble first becomes stable. As can be seen, the solid line divides the $(\rho, T)$ plane into two regions, the region above the line corresponding to stability. From the figure it would seem to be indicated that $\mu / \mu_{\mathrm{f}}=$ constant $\sim 2 \times 10^{-2}$ describes the onset of localization. Thus the mobility decrease up to this point must be described by a fluctuation-type theory, and beyond this point the mobility is governed mainly by well-defined localized states.

Figure 6 shows that the major shortcoming of equation (8) is that the variation of mobility of the quasi-free electron is not properly accounted for. Eggarter's (1972) theory is a quite successful attempt at accounting for the behaviour of the quasi-free electron. That theory is based upon fluctuations in the gas density. Our discussion has ignored this vital physical circumstance. However, as can be seen from the tables the localized state develops a binding energy considerably larger than $k T$ quite rapidly and thus becomes uninfluenced by fluctuations. This is in contrast to Eggarter's notion of pseudo-bubbles, the density of which is obtained from percolation theory rather than thermodynamic stability arguments. Fluctuations will play a role, however, since $\Delta F$ is such a strong function of density that the bubble mobility should certainly be written as an average over the effective radius of the localized state. If the present model of the localized state is to be included into Eggarter's theory we find that the choice of cell size within which fluctuations are to be considered has to be somewhat larger than used by Eggarter (1972).

Another aspect of the problem which can possibly be treated by the models here advanced is the calculation of the lifetimes entering into equation (8). The expression for $\Delta F$ has both a maximum and a minimum. Thus the rate at which an electron localizes itself can be written as

$$
\Gamma=\Gamma_{0} \exp \left(-\Delta F_{\max } / k T\right)
$$

We would expect that $\Gamma_{0} \sim\left(v_{\mathrm{s}} / r_{0}\right)$ where $v_{\mathrm{s}}$ is the velocity of sound. This gives $\Gamma_{0} \sim 5 \times 10^{11} \mathrm{~s}^{-1}$, in quite good accord with values found in the liquid (Onn and Silver 1969). The exact value of $\Delta F_{\max }$ depends upon the way in which the localized state develops. It is entirely possible that $\Gamma$ could be of the same order as the transit 
time through the measuring apparatus. This also is a possible explanation of the results of Harrison and Springett (1971a, b).

We point out that the measurements we present here cover a large range in the $(\rho, T)$ plane and thus can be used as a fairly strong test of any additional theories of the collapse that are advanced. In addition, further experiments could be performed to shed light on this problem. We feel that extensive measurements of the high electric field drift velocity could throw a light on the problem (Levine and Sanders 1967, and Hernandez 1972, private communication), because the quasi-free electron will be much more strongly affected by the field than the bubble. Thus, using a single-gate technique such as we did, when the lifetime of the electron in the quasi-free state is much longer than its transit time through the gate anomalous signals should appear. At low densities we did in fact see anomalous signals but because we were using a $\beta^{-}$source and because the anomalies only occurred at quite low densities it is likely they were due to short-lived $\mathrm{He}^{-}$ions.

Another experimental test which should prove interesting would be to dope $\mathrm{He}$ with $\mathrm{Ne}$, thus reducing the effective $V_{0}$ for a given $\rho$ and $T$ in a known manner. This would provide tests of both Eggarter's (1972) theory and the present localization model.

\section{Acknowledgments}

Several of our colleagues gave us the benefit of useful discussions, particularly V K Wong, $\mathrm{H}$ Gould, and T M Sanders (who suggested using the model potential of equation (6)). We had an illuminating conversation with $\mathrm{M} \mathrm{H}$ Cohen. We would like to thank $\mathrm{J}$ Janecke for help with the computer programs. We wish also to thank J Hernandez for an instructive correspondence.

This research was supported in part by the US Atomic Energy Commission and in part by the Horace H Rackham School of Graduate Studies.

One of us (BES) was supported by an F G Cottrell grant from the Research Corporation.

\section{References}

Clark R C 1965 Phys. Lett. 16 42-3

Eggarter T P 1972 Phys. Rev. A5 2496-509

Eggarter T P and Cohen M H 1970 Phys. Rev. Lett. $25807-10$

- 1971 Phys. Rev. Lett. 27 129-32

Grunberg R 1968 Z. Naturf. 23 1994-2001

Harrison H R 1971 Thesis, University of Michigan (University Microfilms, Ann Arbor, Michigan 48106)

Harrison H R and Springett B E 1971a Phys. Lett. 35A 73-4

-_- 1971 b Chem. Phys. Lett. $10418-21$

Jortner J, Kestner N R, Rice S A and Cohen M H 1965 J. chem. Phys, 43 2614-25

Kuper C G 1959 Phys. Rev. 122 1007-11

Landau L D and Lifshitz E M 1958 Quantum Mechanics: Non Relativistic Theory (New York: AddisonWesley) pp 69-70 108-109

Levine J L and Sanders T M 1967 Phys, Rev. $154138-49$

Neustadter H E and Coopersmith M H 1969 Phys. Rev. Lett. 23 585-9

O'Malley T F 1963 Phys. Rev. $1301020-9$

Onn D G and Silver M 1969 Phys. Rev. 183 295-307

Springett B E, Cohen M H and Jortner J 1967 Phys. Rev. $159183-90$

Springett B E, Jortner J and Cohen M H 1968 J. chem. Phys. 48 2720-31 